\title{
\begin{tabular}{l|l} 
POLITIQUES \& & Politiques et management public
\end{tabular}

\section{La régulation de l'action des enseignants par les chefs de leur établissement}

Regulation of the activity of teachers by their principals

\section{Mohammad Hassani et Denis Meuret}

\section{(2) OpenEdition}

\section{Journals}

Édition électronique

URL : http://journals.openedition.org/pmp/2963

DOI : $10.4000 / p m p .2963$

ISSN : 2119-4831

Éditeur

Institut de Management Public (IDPM)

\section{Édition imprimée}

Date de publication : 15 septembre 2010

Pagination : 103-126

ISSN : 0758-1726

\section{Référence électronique}

Mohammad Hassani et Denis Meuret, «La régulation de l'action des enseignants par les chefs de leur établissement », Politiques et management public [En ligne], Vol 27/4 | 2010, mis en ligne le 15 septembre 2012, consulté le 19 avril 2019. URL : http://journals.openedition.org/pmp/2963 ; DOI : 10.4000/pmp.2963 


\title{
LA RÉGULATION DE L'ACTION DES ENSEIGNANTS PAR LES CHEFS DE LEUR ÉTABLISSEMENT
}

\author{
Mohammad HASSANI* \\ Denis MEURET**
}

\begin{abstract}
Résumé
La régulation de l'action des enseignants par leur chef d'établissement est étudiée à partir d'une enquête auprès des chefs d'établissement scolaire du second degré. II en ressort que les chefs d'établissement jugent en grande majorité légitime d'intervenir sur le travail des enseignants mais qu'un tiers au moins intervient réellement dans ce domaine, de façon plutôt informelle, et que c'est dans leurs établissements, que les enseignants travaillent le plus ensemble.
\end{abstract}

Mots-clés

Régulation, enseignants, chefs d'établissement scolaire, autonomie des établissements scolaires, professionnalité.

\section{REGULATION OF THE ACIVITY OF TEACHERS BY THEIR PRINCIPALS}

Abstract

The regulation of the activity of the teachers by their principals is investigated through a survey of the latter. It shows that principals generally believe that they are entitled to regulate this activity. However, only a third is really engaged in such a regulation, most of the time informally. In their schools, teachers work more together than in the others.

Keywords Regulation, teachers, school autonomy, principals, professionalism.

*Université de Urmia, Iran

**Université de Bourgogne, Institut Universitaire de France - denis.meuret@wanadoo.fr 
Autonomie des établissements, régulation et professionnalité des enseignants
L'autonomie des établissements scolaires s'accroit dans presque tous les systèmes scolaires des pays développés depuis maintenant une trentaine d'années (Meuret, Broccholichi et Duru-Bellat, 2001). En conséquence, la responsabilité et le pouvoir des chefs d'établissement augmentent aussi. On attend d'eux qu'ils obtiennent davantage d'efficacité de leurs enseignants et, par ce moyen, de meilleurs apprentissages pour leurs élèves. Autrement dit, on attend d'eux une intervention plus importante dans la régulation de l'action de leurs enseignants. Dans le contexte français, une première question se pose, qui est simplement la forme et l'ampleur de cette intervention, à la fois parce que l'autonomie des établissements y a augmenté de façon plus " retenue » que dans d'autres pays (Meuret \& Duru-Bellat, 2003) et parce que, dans la tradition française, l'enseignant est redevable à son inspecteur et pas à son chef d'établissement (OCDE, 2007).

Une autre question, liée à la première, est celle des conséquences de l'intervention des chefs d'établissement dans la régulation des pratiques de leurs enseignants sur l'autonomie et le professionnalisme de ces derniers. De cette intervention, en effet, certains redoutent une déprofessionnalisation des enseignants (Meirieu et Lessard, 2005), tandis que d'autres y voient l'occasion d'une professionnalité renouvelé (Pochard, 2008). Nous traitons ces deux questions à partir d'une interrogation de chefs d'établissements du second degré, interrogation à la fois sur leurs pratiques de régulation des enseignants et sur les pratiques de coopération de leurs enseignants. Nous précisons les questions posées (1) avant de répondre à la première par une analyse des résultats de notre enquête (2), puis à la seconde par l'élaboration d'une typologie des établissements (3) et de proposer une discussion de nos résultats (3).

Le schème majeur de la littérature sur l'organisation des établissements scolaires est la tension entre leur aspect bureaucratique et leur aspect professionnel (Bidwell, 1965). La tension entre ces deux aspects est d'autant plus aiguë que chacun s'ancre dans des caractéristiques fondamentales de l'école. D'une part, l'éducation d'un enfant est un processus long, qui fait intervenir de nombreux acteurs et qui vise des objectifs multiples. II en résulte qu'il faut coordonner l'action de nombreux acteurs, d'où la bureaucratie. D'autre part, cependant, la « technologie éducative » exige une interaction persistante entre un enseignant et un élève. Cette interaction permet un attachement minimal de l'élève au maître, sans lequel l'enseignement n'est pas possible ; elle joue un rôle important dans le processus de socialisation lui-même, mais aussi permet à l'enseignant d'évaluer les petites variations qui affectent la performance de l'élève et d'ajuster ses méthodes d'enseignement en conséquence. Les liens interpersonnels qui se nouent au cours de périodes longues fournissent à l'enseignant des ressources affectives puissantes et utiles pour motiver les élèves et donner une certaine force à ses sanctions. En d'autres termes l'enseignement est une activité qui appelle à la fois une coordination fine et durable, sur un temps éclaté en années scolaires, entre plusieurs acteurs, qui nouent chacun une relation forte et intime avec les élèves. En bref, la tension entre les exigences bureaucratiques 
et l'autonomie des enseignants nait d'une tension fondamentale entre une nécessaire division du travail, qui appelle une coordination, et la nature privée (enfermée dans la classe), voire intime (l'enseignant et l'élève sont au fond les seuls à savoir ce qu'ils se font l'un à l'autre) de ce qui doit être coordonné.

Cette tension existe aussi, par exemple, dans les hôpitaux, auxquels on sait que Mintzberg (1982) applique aussi la notion de «bureaucratie professionnelle", mais elle se complique dans le cas de l'école du fait qu'il n'est pas sûr que les enseignants forment une " profession »au sens que la sociologie donne à ce terme. Les enseignants eux mêmes voient souvent leur métier comme l'exercice d'un art, comme la maîtrise des idiosyncrasies subtiles qui se produisent dans les interactions entre les élèves et eux (Chatel, 2002) davantage que comme la mise en œuvre de protocoles diversifiés, à l'instar des médecins. Cette conception de leur métier les protège moins de l'autorité hiérarchique dans leur travail. Elle génère une inquiétude plus grande d'occuper une position subordonnée et aussi deux discours très contrastés sur ce que feraient les enseignants en l'absence de régulation externe. Selon le premier, l'intervention de la bureaucratie met à mal un professionnalisme et une coopération optimale (Mc Neil, 2000, Darling-Hammond, 1990), elle empêche les enseignants de mettre en place un enseignement authentique, mine leur moral et les empêche de s'adapter aux particularités de leurs élèves. Une forme radicale de ce discours considère que la notion même de système scolaire implique la standardisation taylorienne d'une activité qui, par sa nature profonde, ne peut pas l'être (Mc Neil, 1986). Selon le second, livrés à euxmêmes, les enseignants se réfugient plutôt dans la routine, l'usage intensif de manuels et de tests, ils s'isolent dans leur classe, bref, deviennent des techniciens dont la tâche est dictée par les « packages » préfabriqués dont ils dépendent (Cooper et al. 2004).

En France, l'organisation du système scolaire a longtemps répondu à cette tension en assurant la coordination par des programmes d'enseignement détaillés et nationaux d'une part, et d'autre part, par la liberté pédagogique des enseignants, liberté à la fois encadrée et garantie par le fait que leur tutelle était exercée, de loin, par l'inspecteur de leur discipline et non, de près, par le chef d'établissement. II en résulte ce que l'on a pu appeler une "culture corporatiste et libertaire" des enseignants, prompts à se solidarisercontre la direction de leur établissement (OCDE, 2007, p.35).

II se trouve cependant que ce dispositif a été mis à mal de multiples façons (Derouet, 1992), dont la plupart se traduisent par une plus grande autonomie des établissements. Une partie des contenus d'enseignement, une série de formes pédagogiques, sont laissées à leur discrétion. Depuis 1986, les établissements du second degré sont dotés de la personnalité morale et juridique. Depuis 1989, ils élaborent eux-mêmes un « projet d'établissement», voté par leur conseil d'administration, qui porte sur plusieurs aspects de leur fonctionnement. Depuis 2003, ils peuvent s'affranchir des règles nationales pour mener des " expérimentations ", pourvu qu'elles soient évaluées. 
Deux théories ont été avancées en faveur de ce type d'évolution. La première met en avant que les meilleures décisions se prennent au plus près des élèves. La seconde repose sur la théorie de l'Agence : l'activité des Agents (les enseignants) sera davantage orientée vers les objectifs du Principal par un chef d'établissement qui peut, d'une part observer de près les conditions d'enseignement, et diminuer ainsi l'asymétrie d'information dont bénéficient les agents (les enseignants), et qui peut, d'autre part, susciter la loyauté des agents davantage qu'une bureaucratie lointaine. La première théorie peut conduire à concevoir les établissements comme une communauté professionnelle autonome, dans laquelle les décisions sont prises par le collectif des enseignants (Gather Thurler, 2002, Barroso, 2007).

Toutefois, l'évolution réelle des systèmes éducatifs semble avoir plutôt obéi à la seconde, y compris parce que l'autonomie des établissements à elle seule - non encadrée par des objectifs fixés au plus haut niveau dont le chef d'établissement devient le garant et qui accroissent sa légitimité visà-vis des enseignants- n'est pas apparue capable d'améliorer l'efficacité des établissements (Leightwood \& Menzies, 1998). Cette évolution suscite dès lors des critiques qui dénoncent par exemple la contradiction entre la « logique administrative de l'efficience » et le « souci de l'innovation pédagogique " ou encore l'incitation faite aux établissements à chercher des améliorations à court terme, non durables (Lessard\& Meirieu, 2005).

En Angleterre, aux Etats-Unis, ces évolutions, décidées au niveau politiques, se sont traduites par une forte autonomie des établissements accompagnée d'une régulation de leur activité par des dispositifs prégnants et d'une responsabilisation forte du chef d'établissement. En France, portées par la technostructure plus que par les politiques (Meuret, 2007, OCDE, 2007), ces évolutions ont été moins nettes: D'une part le degré d'autonomie atteint par les établissements français, loin d'être négligeable, est bien inférieur à celui qu'ont atteint les établissements anglais, par exemple ${ }^{1}$. D'autre part, les textes juridiques ont accru les prérogatives du chef d'établissement, mais avec des formulations qui ménagent en même temps la «liberté pédagogique » des enseignants. Le "référentiel du métier de chef d'établissement » (2000), stipule que le ce dernier "conduit la politique pédagogique et éducative de l'établissement», "est le garant de l'efficacité pédagogique», ce qui lui donne sur les enseignants une certaine autorité.

Dans le même sens, un rapport de la Cour des Comptes (2007, p. 200) note que « la nouvelle approche, plus solidaire et cohérente, des différentes activités de l'établissement conduit à placer son responsable au centre d'une communauté qu'il lui revient d'animer et de fédérer». Mais, le détail de la rubrique «conduit la politique pédagogique » dans le référentiel met

\footnotetext{
${ }^{1}$ Les établissements du second degré inférieur prennent de façon autonome 39\% des décisions qui affectent leur fonctionnement, contre $91 \%$ en Angleterre (OCDE, 2008, indicateurD6.1).
} 
l'accent sur la périphérie de l'enseignement (l'orientation des élèves, leur évaluation, leur assiduité) et non sur son cœur. Ledit référentiel stipule aussi « la collaboration du chef s'établissement avec les corps d'inspection permet d'assurer la qualité des enseignements", ce qui retire au chef d'établissement une partie de l' l'autorité qui lui est dévolue par ailleurs. Illustration de cette autorité partagée, le chef d'établissement attribue chaque année à chaque enseignant une "note administrative " qui pèse $40 \%$ de sa note finale tandis que la «note pédagogique » de l'Inspecteur pèse $60 \%$. Autrement dit, la régulation des enseignants - au sens étroit que nous utilisons ici : l'ensemble des actions de leur tutelle qui visent à faire en sorte qu'ils poursuivent efficacement les objectifs du système scolaire- est partagée entre le chef d'établissement et l'Inspecteur. Plus précisément, le chef d'établissement a en charge la coordination entre les enseignants, mais sa légitimité à intervenir sur les pratiques de chacun d'eux reste très faible. Or, l'efficacité d'un établissement se décide d'abord dans chacune de ses classes (Grisay, 1997).

Le contexte juridique met donc des limites assez étroites à la régulation du travail des enseignants par le chef de leur établissement. Le contexte politique, lui, lui est nettement défavorable : "Toute tentative pour donner plus de responsabilité au chef d'établissement est dénoncée par le syndicat (des enseignants) majoritaire comme attentatoire à la liberté pédagogique, à l'égalité de traitement, à lindépendance du jugement des inspecteurs et comme dissimulant (...) une volonté de mise au pas par les "petits chefs" (OCDE, 2007, p36). Toutefois, l'attachement de ce même syndicat aux programmes rigides du modèle traditionnel ouvre aux chefs d'établissement la possibilité de justifier leur intervention sur le travail des enseignants au nom de l'adaptation de l'enseignement aux besoins des élèves. De fait, on trouve dans leur discours l'idée qu'ils portent le souci de cette adaptation, contre des enseignants décrits comme n'en étant pas assez soucieux et, souvent, trop durs et impersonnels avec les élèves (Grellier, 1997, Barrère, 2006).

Le premier objet de ce travail est de cerner quelle représentation, dans ce contexte, les chefs d'établissement se font de leur légitimité à intervenir sur le travail des enseignants et quel type de régulation de ce travail ils mettent en œuvre. On peut émettre l'hypothèse d'une régulation plutôt informelle et portant plutôt sur la périphérie de l'enseignement que sur les modalités de l'enseignement en classe.

Le second est de tenter de repérer l'impact de cette intervention sur la professionnalité des enseignants, en appelant ainsi un certain degré d'autonomie et d'expertise qui est la marque des «professions ». On l'a vu, cette question n'est pas nouvelle : l'idée que l'autonomie des enseignants est mise à mal par la régulation était émise aussi contre l'ancienne régulation, bureaucratique. Sa forme actuelle, en France, est de savoir si, du point de vue de leur professionnalité, les enseignants gagnent ou perdent à exercer leur métier dans des établissements plus autonomes, dans un cadre plus souple, sur lequel ils peuvent davantage influer, mais où ils sont davantage exposés à la pression de leur chef d'établissement. On l'a vu, l'intervention de 
ce dernier est, pour certains, nuisible à la professionnalité des enseignants: c'est la théorie du « petit chef », de la « prolétarisation » (McNeil, 2000), c'est l'incompatibilité entre le temps court de l'administration moderne et le temps long des actions pédagogiques durables, entre " l'efficience administrative " et " l'innovation pédagogique ». Les sociologues anglais associent aussi à l'autonomie des établissements et à l'environnement administratif qui l'accompagne « une escalade, pilotée depuis les bureaux, de pressions, d'exigences (expectations) et de contrôles sur ce que font les enseignants et sur combien ils devraient faire pendant leurs journées d'enseignement " (Hargreaves, 1994, cité par Hassani, 2007). Comme la régulation des enseignants par le chef d'établissement est un des éléments d'un programme à l'œuvre dans tous les pays européens et dont le modèle est anglais (Barroso, 2007), les enseignants français seraient menacés, comme leurs collègues anglais, d'une dépersonnalisation du métier, d'une perte d'autonomie, d'un manque de temps pour le développement des compétences, d'une surcharge chronique de travail, du remplacement du temps passé avec les élèves par la réponse aux demandes administratives (Ball, 2003). Alors que les chefs d'établissement, sont « les héros de la nouvelle régulation» (Maroy, 2006, p. 228) les enseignants assisteraient à une « érosion de (leur) autonomie professionnelle» (id., p.354).

Ceux pour qui l'autonomie des établissements-y compris accompagnée d'une pression accrue du chef d'établissement- est favorable à la professionnalité des enseignants- à leur expertise et à leur autonomie- mettent en avant que le chef d'établissement intervient comme porteur d'un « projet d'établissement " élaboré collectivement. Pour eux, autonomie des établissements, logique de projet, travail collectif, vont ensemble et vont avec l'évolution qui fait des enseignants des « praticiens réflexifs » (Tardif et Lessard, 2005), capables de modifier leurs pratiques en fonction de l'évaluation des performances de leurs élèves et de leur établissement, une figure plus professionnelle que celle de l'enseignant routinier de la régulation bureaucratique. Toujours pour eux, la régulation des enseignants par le chef d'établissement, dans la mesure où elle est au service de l'apprentissage des élèves, ne peut que renforcer une professionnalité qui poursuit le même objectif.

Nous nous demanderons si la professionnalité des enseignants est plus (ou moins) forte dans les établissements où leur régulation par le chef d'établissement est plus forte. Nous apprécierons, on y reviendra, cette professionnalité par la fréquence avec laquelle ils se réunissent entre pairs pour des motifs professionnels. Ce critère est partiel mais il a l'avantage d'être lié aux deux dimensions de la professionnalité puisque prendre ensemble des décisions professionnelles renforce l'expertise des participants en même temps que c'est un signe de leur autonomie. Par ailleurs, d'une part c'était le seul critère accessible par une enquête auprès des chefs d'établissement, d'autre part il permet de mesurer si l'individualisme pédagogique du modèle traditionnel est remplacé, là où les chefs d'établissements s'autorisent davantage à intervenir sur le travail de leurs enseignants, par un individualisme soumis ou par une appropriation collective des marges de manœuvre offertes par la plus grande autonomie 
des établissements. Nous adjoignons par ailleurs à la mesure de l'intensité de la régulation des enseignants par le chef d'établissement une appréciation de ses pratiques de pilotage ${ }^{2}$, en particulier à travers son usage des indicateurs que l'administration met à sa disposition pour piloter son établissement. En principe, l'usage de ces indicateurs est, comme la régulation des enseignants, un indice de la volonté d'un chef d'établissement de tirer parti de l'autonomie de son établissement pour améliorer le service rendu aux élèves (OCDE, 2007).

Ce questionnement est la source d'une enquête que nous avons menée en 2005, par questionnaire, auprès des huit cent six chefs d'établissements publics et privés du second degré des académies de Bordeaux et Dijon. Elle faisait partie du travail réalisé pour une thèse soutenue par $M$. Hassani et dirigée par D. Meuret.

\section{L'investigation mise en œuvre}

Quatorze journées de shadowing - où l'observateur suit comme son ombre le sujet dont il veut étudier la pratique - de sept chefs d'établissement, soit l'observation de leurs interactions quotidiennes avec les enseignants, ont aidé à concevoir un premier questionnaire de 70 questions, ramené à 40 après qu'il ait été soumis à six autres chefs d'établissement de l'académie de Bourgogne et au responsable de la formation des chefs d'établissement au rectorat de Dijon.

Ces 40 questions appelaient le plus souvent une réponse sur une échelle de type Likert. Elles portaient sur les normes professionnelles des chefs d'établissement et surtout sur leurs pratiques. Ces dernières ont été scrutées dans deux dimensions : le degré d'intervention auprès des enseignants surtout et aussi leur usage des indicateurs. Un second ensemble de questions portait sur la coopération entre les enseignants.

Un risque de ce genre d'enquête est d'obtenir des réponses entachées de biais de désirabilité, par exemple une surestimation du « travail en équipe " des enseignants, dans la mesure où cette pratique est valorisée par la technostructure du ministère. Nous avons tenté de diminuer ce risque en posant des questions précises sur la fréquence des pratiques, des chefs d'établissement comme des enseignants.

Trois cent quatorze chefs d'établissement ont répondu à l'enquête. Le taux de réponse est donc de $39 \%$, un peu plus élevé pour les collèges et lycées professionnels (41\%) que pour les lycées d'enseignement général et technologiques (29\%), plus élevé aussi dans l'enseignement public $(46 \%)$ que dans l'enseignement privé (22\%).

\footnotetext{
2 « pilotage» est le mot utilisé au sein de l'éducation nationale pour désigner le management des établissements (OCDE, 2007).
} 


\section{La régulation des enseignants : normes et pratiques des chefs d'établissement}

Nous utilisons ci-dessous cette enquête pour répondre aux deux questions exposées.

\section{Les normes professionnelles des chefs d'établissements}

La quasi totalité des répondants ${ }^{3}$ estime que «c'est d'abord le travail des enseignants qui fait l'efficacité de l'établissement» et considère que les enseignants aiment être responsables et autonomes dans leur travail. Pour eux, cependant, la coordination des enseignants ne peut plus reposer sur les moyens traditionnels, les programmes : seuls, un très petit nombre de répondants $(4 \%)^{4}$ estime que les réunions entre enseignants sont une perte de temps et la quasi-totalité, à nouveau, estime que le travail en équipe " ajoute beaucoup à l'efficacité de chaque enseignant ».

Bien que conscients de l'attrait de l'autonomie pour les enseignants, les répondants ont une conception clairement interventionniste de leur rôle: $97 \%$ estiment qu'ils « doivent jouer un rôle pédagogique " et, plus précisément, " estiment légitime de contrôler eux même la qualité du travail des enseignants ». Plus significatif encore, $45 \%$ seulement des répondants estiment que les enseignants d'une discipline doivent toujours avoir le dernier mot sur sa pédagogie et $78 \%$ estiment que « la direction et la supervision des enseignants font partie de leur tâche ».

Une forte majorité des chefs d'établissement interprète donc de façon large les responsabilités que leur accorde le référentiel de leur métier et tient pour légitime d'intervenir, non seulement pour coordonner le travail des enseignants, mais pour contrôler le travail de chacun d'eux. S'il est vrai que les chefs d'établissement anglais sont «les héros» de la régulation moderne (Maroy, 2006), les réponses à notre questionnaires indiquent qu'une majorité des chefs d'établissement français est prête à jouer un tel rôle, bien que leurs pratiques, on va le voir, soient moins fréquemment interventionnistes que ce que suggèrent leurs normes professionnelles. Contrairement à ce qu'on aurait pu attendre, les normes professionnelles des chefs d'établissements de l'enseignement privé et de l'enseignement public sont proches. Par exemple, ils sont $79 \%$ dans le public et $84 \%$ dans le privé à estimer que l'une des tâches du chef d'établissement est la direction et la supervision du travail des enseignants (Hassani, 2007, p 172).

\footnotetext{
${ }^{3}$ On trouvera en annexe 1 les questions posées aux chefs d'établissement et leurs réponses.

${ }^{4} \mathrm{Si}$ on estime, pour se prémunir d'éventuels biais de désirabilité, que la proportion des réponses indiquant coopération des enseignants et intervention du chef d'établissement dans leur régulation est deux fois plus élevée parmi les répondants que parmi les non répondants, la fréquence des réponses de ce type parmi les chefs d'établissement doit être estimée à 0695 fois celle qui prévaut parmi les répondants (puisque le taux de réponse est 0,39 , si $x$ est la fréquence de la réponse parmi les répondants, la fréquence parmi les enquêtés est $y=0,39 x+(1-0,39)(x / 2))$. La même règle conduirait à estimer que la fréquence des réponses anticoopératives et bureaucratiques (ex : les réunions d'enseignants sont une perte de temps) doit être estimée à 1,39 fois celle qui prévaut parmi les répondants. Toutefois, compte tenu du caractère très hasardeux de ce redressement, nous donnons dans le texte les fréquences brutes, c'est-à-dire parmi les répondants.
} 


\section{Les pratiques de régulation des enseignants}

93\% des chefs d'établissement qui ont répondu au questionnaire «rencontrent les enseignants de façon informelle» souvent ou assez souvent. Ce qu'on pourrait appeler la conception jupitérienne d'un chef d'établissement isolé sur l'Olympe de son administration ou uniquement préoccupé des relations publiques de son lycée est devenue très minoritaire. Cela se voit aussi au fait que les trois quarts de nos répondants disent être intervenus l'an dernier dans la classe d'un de leurs enseignants à propos d'un problème rencontré par ce dernier et que les deux tiers sont entrés au moins trois fois, l'an dernier, dans la classe d'un de leurs enseignants ${ }^{5}$.

Trois quart des répondants disent «rencontrer individuellement les enseignants pour évoquer les performances des élèves ", et la moitié dit le faire plus d'une fois par an. II s'agît là d'une démarche relativement formelle, mais il semble qu'au cours de la plupart de ces rencontres, comme au cours des rencontres informelles évoquées précédemment, soit contournée la responsabilité des enseignants dans les performances de leurs élèves : ce ne sont que $45 \%$ de nos répondants qui disent « discuter souvent ou assez souvent avec les enseignants des points faibles et des points forts de leur travail ». Autrement dit, alors que la grande majorité des chefs d'établissement parle souvent avec ses enseignants, que les trois quarts évoquent avec eux les performances de leurs élèves, une petite moitié seulement aborde la question de la responsabilité des enseignants eux-mêmes dans ces performances. Ce n'est pas anecdotique, mais ce n'est pas fréquent. De même, si la relative fréquence de l'entrée du chef d'établissement dans les classes semble témoigner, à son tour, de ce que Barrère (2002) appelle «l'entrouverture » des classes, les réponses aux autres questions indiquent que ce comportement n'implique le plus souvent aucun jugement sur le travail de l'enseignant.

Enfin, il est rare qu'un chef d'établissement conseille à un enseignant de suivre telle ou telle formation continue (moins de $20 \%$ des répondants disent le faire souvent ou assez souvent), alors qu'on peut considérer qu'il s'agît d'une façon particulièrement positive et aidante d'attirer l'attention sur une insuffisance. Autre indice de la relative faiblesse des pratiques de régulation formelle, seulement $27 \%$ des répondants disent «indiquer formellement aux enseignants les critères selon lesquels ils jugent de la qualité de leur travail ». Notre enquête, après d'autres (Barrère, 2006, van Zanten et al, in Maroy, 2006) montre que les chefs d'établissements doivent compter

\footnotetext{
${ }^{5}$ Ces interventions dans les classes sont sans doute consacrées la plupart du temps à soutenir l'enseignant vis à vis de ses élèves, une tâche qu'on peut ne pas assimiler à de la régulation, mais qui lui est sans doute associée : si un enseignant a recours au soutien du proviseur, il est plus accessible aux avis de ce dernier sur son travail. Nous avons posé cette question parce que, dans une étude sur l'efficacité des collèges, la seule caractéristique du chef d'établissement liée à l'efficacité de son collège était précisément la fréquence avec laquelle il entrait dans les classes (Grisay, 1997).
} 
sur le charisme, la suggestion, bref l'informel, pour exercer une régulation qu'ils situent pourtant au cœur de leurs priorités. Mais elle montre aussi que l'hypothèse d'une régulation purement informelle et portant seulement sur des activités périphériques (les relations avec les parents, par exemple) n'est vérifiée que pour un faible nombre de chefs d'établissement. Selon les autres, la régulation de l'activité des enseignants, sans être invasive, existe et, point essentiel, l'enjeu en est bien d'améliorer la progression des élèves.

On peut estimer qu'il s'agît là des établissements dont les chefs estiment forte ou assez forte leur action sur l'enseignement, respectivement 6 et $58 \%$ des répondants. Les chefs des établissements privés interviennent un peu plus pour réguler l'activité de leurs enseignants que leurs homologues du public, mais la différence est, ici encore, moindre qu'on aurait pu le croire. Soit les deux critères probablement les plus significatifs d'une régulation forte : $58 \%$ des répondants dans le privé et $41 \%$ dans le public « discutent souvent ( $16 \%$ vs $15 \%$ ) ou assez souvent ( $42 \%$ vs $26 \%$ ) avec les enseignants des points faibles et forts de leur travail »; $19 \%$ dans le privé et $16 \%$ dans le public « conseillent souvent ou assez souvent à un enseignant de suivre telle formation continue » (Hassani, 2007, p. 163-165). Cette proximité est un autre indice de ce que les chefs d'établissements du public interviennent de façon significative pour réguler l'activité de leurs enseignants.

\section{La coopération entre enseignants}

Barrère (2002) a donné, d'après une enquête par entretiens après d'une quarantaine d'enseignants de la région du Nord, un diagnostic nuancé sur la fréquence du travail collectif des enseignants : non point absent, puisque pratiqué par une petite moitié des interrogés, mais informel et affinitaire, rarement très organisé et très durable, pas forcément très prenant. Notre enquête donne un tableau un peu plus positif, du moins si on estime positif le travail collectif des enseignants. Selon $86 \%$ des répondants " tous comptes faits, le travail collectif existe dans mon établissement ». De façon plus concrète, selon $66 \%$ des répondants, les enseignants d'une même discipline coopèrent de façon significative (se réunissent au moins trois fois par an), et, selon $50 \%$, c'est le cas des enseignants d'une même classe (ils se réunissent au moins trois fois par an en dehors des conseils de classe).

Le travail collectif des enseignants ne semble donc plus réservé, dans une bonne moitié des établissements, à l'organisation du voyage annuel ou à des projets ponctuels, il porte sur le cœur de l'activité des enseignants et non sur sa périphérie : selon $75 \%$ des répondants, les enseignants de leurs établissements " discutent souvent ou assez souvent entre eux des performances de leurs élèves et des moyens de les améliorer "; selon 38\% des répondants, les "enseignants d'une même discipline se coordonnent " souvent ou assez souvent " sur les objets ou les méthodes de l'évaluation. Ensuite, il prend assez souvent, on vient de le voir, une forme systématique. Enfin, les enseignants ne se contentent pas de parler ensemble : selon $51 \%$ des répondants, les enseignants prennent "souvent ou assez souvent, ensemble des décisions pédagogiques ». 


\section{Pratiques de pilotage des établissements}

$59 \%$ des répondants disent réunir les enseignants plus de deux fois par an pour parler « du projet d'établissement ou de l'établissement en général ", ce qui peut être considéré comme un mode de travail collectif des enseignants, une pratique de régulation des enseignants et une pratique de pilotage. II semble que les indicateurs que l'administration met à la disposition des établissements, et qui permettent à chacun de comparer les conditions de l'enseignement et ses résultats avec ceux qui prévalent dans les autres, soient assez peu mobilisés au cours de ces réunions. En effet, si une moitié des répondants déclarent utiliser régulièrement les indicateurs que leur envoie l'Administration Centrale, seulement 22\% disent en discuter avec certains enseignants.

Cette enquête indique une plus forteintervention des chefs d'établissement dans la régulation des enseignants et une plus forte coopération entre les enseignants que les enquêtes, plus anciennes, qui procèdent d'une interrogation des enseignants (Barrère, 2002, questionnaire aux enseignants de Grisay, 1997, Lang, 1999), même si elle confirme la distance considérable que Maroy (2006) donne à voir à cet égard entre l'Angleterre et la France. Pour fixer les idées, on peut estimer, en utilisant la prudente règle de redressement proposée en note 4, qu'au moins les deux tiers des chefs d'établissement ont une représentation de leur métier qui les autorise à intervenir de façon significative auprès des enseignants pour améliorer les progrès des élèves, qu'un tiers d'entre eux intervient de façon assez significative auprès de leurs enseignants pour réguler leur activité, qu'un tiers encore constate une coopération significative entre les enseignants de son établissement.

Ces réponses peuvent témoigner de paysages profondément différents, selon la façon dont ces tiers se combinent. $\mathrm{Si}$, par exemple, les chefs d'établissement qui déclarent agir pour réguler l'activité de leurs enseignants sont ceux qui déclarent que ces derniers se coordonnent, les résultats confortent plutôt le discours officiel, selon lequel la nouvelle régulation favorise l'émergence d'une nouvelle professionnalité. $\mathrm{Si}$, en revanche, ce ne sont pas les mêmes, notre enquête confortera le discours critique, qui, lui, oppose une logique de type autogestionnaire (les enseignants se coordonnent sans nul besoin de l'administration) à la logique de l'administration (la pression mises par les chefs d'établissement au nom de l'administration empêche les enseignants de faire convenablement leur travail au service des élèves). C'est ce paysage que la suite de l'analyse

Modes de régulation et modes de coordination, nouvelle professionnalité ou prolétarisation? cherche à faire apparaître.

Nous avons cherché à définir des modes de régulation à partir des correspondances observables entre les diverses réponses au questionnaire, au moyen d'une analyse factorielle des correspondances, sur les premiers facteurs de laquelle a été pratiquée une classification hiérarchique. Cette classification a été arrêtée à quatre groupes que nous désignons par deux qualificatifs, dont le premier vise la coopération entre les enseignants et 
le second l'intervention du chef d'établissement dans la régulation de leur travail. L'annexe 2 représente les projections sur le plan défini par les deux premiers axes de l'analyse, dans lequel apparaissent les quatre types d'établissements.

Dans les établissements de type 1 (29,5\% des répondants), l'activité collective des enseignants est plus forte qu'ailleurs de même que la régulation des enseignants par le chef d'établissement. Les enseignants discutent beaucoup du niveau de performance des élèves et des moyens de les améliorer, les enseignants d'une même discipline ou d'une même classe se réunissent au moins quatre fois par an, ceux d'une même classe se coordonnent souvent, notamment sur l'objet ou les méthodes d'évaluation, ils prennent souvent ensemble des décisions pédagogiques. Les chefs d'établissement rencontrent souvent de façon informelle les enseignants, réunissent les enseignants au moins quatre fois par an pour parler du projet d'établissement ou de l'établissement en général, discutent avec les enseignants des points forts et faibles de leur travail, rencontrent individuellement les enseignants pour discuter des performances de leurs élèves, conseillent aux enseignants des formations continues. En moyenne, ces chefs d'établissement rencontrent les enseignants dans la salle des professeurs quatre fois ou plus par semaine. Ils contrôlent les cahiers de texte des enseignants et considèrent que les enseignants de leur établissement travaillent de façon collective. Ils utilisent régulièrement les indicateurs académiques.. Dans ces établissements au moins, le discours officiel sur la convergence d'une nouvelle professionnalité et d'une intervention du chef d'établissement dans la régulation des enseignants est vérifié. $\mathrm{Ce}$ type d'établissement peut être appelé «fortement collectif et fortement régulé». S'agissant des modalités du pilotage, les chefs d'établissement de ce groupe utilisent les indicateurs académiques régulièrement mais pas forcément les indicateurs IPES, envoyés par l'administration centrale.

Les établissements de type 2 (15,4\% des répondants) sont à l'opposé du type 1 , et vérifient donc aussi à leur façon le discours officiel moderniste. Ce sont des établissements où il y a peu d'activité collective des enseignants et peu d'intervention du chef d'établissement sur ses enseignants. Les chefs d'établissement de ce groupe ne contrôlent pas le cahier de texte des enseignants, n'interviennent pas dans les classes en cas de problème rencontré par l'enseignant. Ils n'entrent jamais dans les classes de façon rapide et informelle, ne rencontrent pas individuellement les enseignants pour discuter des performances de leurs élèves. Par ailleurs, dans les établissements de ce groupe, les enseignants d'une même classe ou d'une même discipline se réunissent seulement une fois par an. Ce type de fonctionnement peut être appelé : « Individuel/ laissez -faire». Les chefs d'établissement de ce groupe n'utilisent pas non plus les indicateurs mis à leur disposition pour piloter leur établissement.

Le type 3 concerne $48,3 \%$ des répondants, c'est le plus fréquent. Ici, les activités collectives des enseignants existent, mais sont moins fréquentes que dans les établissements du type 1 et les interventions 
des chefs d'établissement sont plus modérées : réunion des enseignants d'une même discipline ou d'une même classe en moyenne 2 fois par an, coordination entre les enseignants d'une même discipline et d'une même classe sur l'objet ou les méthodes d'évaluation quelques fois seulement. Les chefs d'établissement discutent «quelquefois» avec les enseignants des points forts et faibles de leur travail. Les enseignants prennent "quelquefois» ensemble des décisions pédagogiques. En moyenne, les chefs d'établissement réunissent les enseignants pour parler du projet d'établissement ou de l'établissement 2 fois par an. Le chef d'établissement conseille «quelquefois» les enseignants par rapport à des formations continues. Certaines pratiques informelles sont assez fréquentes: les enseignants discutent assez souvent entre eux du niveau de performance des élèves et des moyens de l'améliorer, ceux d'une même classe se coordonnent assez souvent entre eux. Ce type de régulation, qui prévaut donc dans la moitié des établissements peut être appelé "moyennement collectif et moyennement régulé». Les chefs d'établissement de ce groupe déclarent plutôt utiliser les indicateurs académiques.

Le type 4 regroupe seulement $6,8 \%$ des réponses. Ce sont des établissements où les activités collectives sont peu fréquentes voire inexistantes. Toutefois deux activités administratives les distinguent des établissements du type 2, comme si le chef d'établissement y était le seul à porter la dimension collective du travail. II n'y a pas de coordination entre les enseignants d'une même discipline sur l'objet ou les méthodes de l'évaluation, les chefs d'établissement déclarent que les enseignants de leur établissement ne partagent pas les mêmes valeurs professionnelles, ceux d'une même classe se coordonnent entre eux rarement et ne se réunissent jamais en dehors des conseils des classes. Selon les chefs d'établissement, le travail collectif n'existe pas dans l'établissement, les enseignants discutent rarement du niveau de performance des élèves et des moyens de les améliorer. Les enseignants prennent rarement des décisions pédagogique ensemble. Le chef d'établissement discute rarement avec les enseignants des points faibles et forts de leur travail. Cependant, les chefs d'établissement rencontrent assez souvent les enseignants pendant la récréation ou à d'autres occasions informelles, et réunissent les enseignants trois fois dans l'année pour discuter de projets d'établissement ou de l'établissement de façon générale. Ce type de régulation peut être appelé «Individuel/ Interventionniste». Le chef d'établissement essaie d'impulser du lien social, mais cette pratique rencontre peu d'écho auprès de ses enseignants.

Le type "fortement collectif et fortement régulé" est un peu plus fréquent dans les collèges que dans les lycées, dans l'enseignement public que dans l'enseignement privé, dans les ZEP que hors ZEP, ce qui confirme le constat que les enseignants coopèrent davantage dans les établissements difficiles (Kherroubi et Van Zanten, 2000). Les enseignants y sont plutôt jeunes, ce qui confirme le constat de Rayou et van Zanten (2004) à partir de leur investigation des attitudes des nouveaux enseignants. Ce sont des établissements de petite taille et les taux de redoublement y sont plutôt faibles. 
Le type «Individuel/Laisser Faire» est un peu plus fréquent en lycée professionnel que dans les collèges et les lycées généraux. Les taux de redoublement y sont plus élevés qu'ailleurs. II est nettement plus fréquent dans le privé que dans le public, ce qui contredit l'image moderniste que l'on a parfois de l'enseignement privé.

Le type «moyennement collectif et moyennement régulé » est un peu plus fréquent en lycée que dans les deux autres catégories, les enseignants y sont âgés.

Le type «Individuel/Interventionniste» est plus fréquent dans les ZEP, comme le type « fortement collectif et fortement régulé».

Cette enquête conforte plutôt la thèse de la nouvelle professionnalité, puisque la coopération entre les enseignants apparaît plus, et non moins, intense là où les chefs d'établissement s'engagent davantage dans la régulation des enseignants. Ces grandeurs, au moins telles que nous les appréhendons, sont fortes ensemble dans les établissements de type1, moyennes ensemble dans les établissements de type3, faibles ensemble dans les établissements de type 2. Elles divergent seulement dans les établissements de type 4, qui représentent seulement $7 \%$ des établissements. En revanche, cette analyse tend à montrer que l'usage des indicateurs, dont les réponses brutes montrent qu'il est assez fréquent, est sans lien avec l'intervention des chefs d'établissement dans la régulation de leurs enseignants, en particulier s'agissant des indicateurs IPES élaborés par l'administration centrale. On aurait pu s'attendre à ce que les chefs d'établissement qui interviennent dans la régulation de leurs enseignants utilisent aussi davantage ces indicateurs, qui contiennent des données en principe utiles dans cette perspective. Cela ne semble pas être le cas.

Discussion

Quels éléments cette enquête apporte-t-elle au débat sur l'effet de la régulation moderne sur la professionnalité enseignante ? Selon Maroy (2005), ce débat met aux prises ce qu'il appelle «le discours modernisateur », lequel associe des enseignants réflexifs, des établissements portés par une dynamique collective à un Etat régulateur et évaluateur, et un « discours critique ", qui associe à cette même régulation plutôt des enseignants prolétarisés et démoralisés par la perte de sens et l'inauthenticité croissante de leur métier (cf. aussi Ball, 2003).

Ce discours modernisateur existe en France, même s'il se traduit moins dans les faits que dans les pays anglo-saxons. Le récent Livre vert sur la condition enseignante (Pochard, 2008), en est un exemple, qui associe, lui aussi, à un chef d'établissement qui « incarne le projet d'établissement (...) signe le contrat d'objectif avec l'autorité de tutelle et (doit) à ce titre être le porteur du processus d'évaluation ", des enseignants capables de dépasser leur ancrage disciplinaire pour coopérer avec leurs collègues au niveau de l'établissement. Pour le discours modernisateur, c'est la recherche de l'efficacité scolaire qui induit ces changements. Au contraire, pour le discours critique, la régulation moderniste est un avatar de l'idéologie néolibérale (Barroso, 2007). Sans égard pour la spécificité de la forme scolaire, cette idéologie plaque sur les systèmes scolaires des recettes 
issues du monde de l'entreprise, de sorte que la nouvelle régulation n'a aucune raison d'être associée à une plus grande coopération entre les enseignants, dont elle va rendre le travail plus intense, plus mécanique, moins gratifiant, et la dépendance à l'égard du chef d'établissement plus étroite. II importe d'ailleurs de noter que ce débat n'est pas limité aux chercheurs en éducation, il traverse le corps enseignant lui-même, du moins en Angleterre (Ball, 2003) et aux Etats-Unis (Louis, 2005) : certains, interrogés sur les conséquences de la nouvelle régulation, optent pour la thèse de la perte d'autonomie tandis que d'autres optent pour celle de la professionnalisation.

En France, il apparaît que les normes professionnelles des chefs d'établissement et des enseignants divergent considérablement. La comparaison entre notre enquête, qui donne le point de vue des premiers, et le sondage commandé par le Syndicat National de l'Enseignement Secondaire (SNES) à l'institut $\mathrm{CSA}^{6}$, qui donne le point de vue des seconds, le montre. A 70\% environ, les enseignants s'y déclarent contre toute déclinaison locale de leurs obligations de service ou des contenus de l'enseignement, contre quelque prérogative que ce soit du chef d'établissement en matière de recrutement, contre toute intervention de ce dernier dans l'évaluation de leur travail, ainsi que contre le fait qu'il nomme les membres du Conseil Pédagogique de l'Etablissement, Conseil dont, à la même proportion, ils récusent toute ingérence dans leur pratique ${ }^{7}$. Autrement dit, en grossissant le trait, $70 \%$ des enseignants sont, d'après ce sondage, opposés à un mode de fonctionnement de l'établissement que $70 \%$ des chefs d'établissement au moins trouvent, selon notre enquête, normal et légitime, et réciproquement ${ }^{8}$, ce qui signifie, en grossissant encore le trait, qu'en appariant au mieux les uns et les autres, on aurait $30 \%$ d'enseignants et de chefs d'établissement à peu près d'accord sur le modèle « individuel laissez faire ", 30 autres \% à peu près d'accord sur le modèle " collectif/ régulé » et $40 \%$ en désaccord avec leurs partenaires respectifs. Au-delà d'une réticence à tout accroissement de l'influence du chef d'établissement

\footnotetext{
${ }^{6}$ Sondage téléphonique auprès de 503 professeurs de l'enseignement secondaire public, réalisé du 25 au 28 mars 2008. Ce sondage, mis en ligne sur le site du SNES le 16.4.08, interroge les enseignants sur certaines recommandations du rapport Pochard.

${ }^{7}$ Le Conseil Pédagogique, créé par la Loi d'orientation de 2005, est composé au moins d'un enseignant par discipline et d'un « professeur principal » par niveau. La circulaire de rentrée 2006 précise ses modalités de fonctionnement : elle laisse chaque établissement libre de choisir la modalité d'élection de ses membres et lui confie la responsabilité du « volet pédagogique du projet d'établissement » et il examine la conformité à ce Projet des expérimentations prévues. Dans le sondage, $63 \%$ des enseignants se déclarent opposés à ce qu'il puisse " prendre certaines décisions d'ordre pédagogiques, qui s'imposent aux enseignants ", autant dire à ce qu'il accomplisse sa tâche.

${ }^{8}$ En particulier, rappelons que $97 \%$ de nos répondants, au moins $67 \%$ des chefs d'établissement, estime " légitime de contrôler eux même la qualité du travail des enseignants ", tandis que, selon le sondage, $73 \%$ des enseignants sont opposés à une « plus grande intervention du chef d'établissement dans l'évaluation des enseignants. "
} 
sur leur travail, ce sondage donne à voir que, toujours à 70\% environ, les enseignants sont persuadés que toute localisation ou personnalisation des décisions qui les concernent se ferait à leur détriment et que l'impersonnalité de la gestion administrative est leur meilleure protection. Cette situation contribue sûrement à expliquer le " malaise " des chefs d'établissement (OCDE, 2007) comme celui des enseignants (Pochard, 2008).

Notre enquête montre donc que le débat sur la professionnalité des enseignants se pose dans des conditions très différentes en France et dans les pays anglo-saxons, qu'il s'agisse de l'Angleterre (Ball, 2003) ou des Etats Unis (Mc Neil, 2000 ; Valencia, 2004). Alors que l'établissement scolaire est, dans la dispute anglo-saxonne, le théâtre d'une concurrence entre le modèle de la communauté professionnelle et celui de la régulation moderniste d'un établissement dont les deux partis souhaitent l'autonomie, selon les enseignants français, du moins tel que ce sondage les donne à voir, tout renforcement de l'autonomie de l'établissement, et bien sûr des prérogatives de son chef, ne peut que se traduire par leur abaissement puisque leur autonomie et leur dignité sont garanties par leur isolement, par la dévotion exclusive à leur cours et au rayonnement de leur discipline.

Peut-on tirer de cette enquête les contours d'une action susceptible d'atténuer le malaise des personnels du système éducatif ? Ce pourrait être une sorte de conjugaison du modèle de la communauté professionnelle et de la régulation par le chef d'établissement. Un cercle vertueux peut s'enclencher, dans lequel le chef d'établissement favorise une coopération entre les enseignants d'où émerge un éthos collectif au nom duquel le chef d'établissement peut réguler l'activité de ses enseignants et ainsi éloigner le spectre du " petit chef » dont les objectifs et l'action apparaissent, soit arbitraires, soit comme une traduction des objectifs de l'administration qui ne tient pas compte de la situation particulière de l'établissement.

\section{BIBLIOGRAPHIE}

Ball, S.J., 2003, The teacher's soul and the terrors of performativity, Journal of Education Policy, 18 (2), 215-228.

Barrère, A., 2006, Sociologie des chefs d'établissement. PUF, Paris.

Barrère, A. (2002) Pourquoi les enseignants ne pratiquent-ils pas le travail en équipe ? Sociologie du Travail, 44, 481-497.

Barroso, J., 2007, L'autonomie et la gestion de l'école publique : entre le marché, le managérialisme et la démocratie, in Derouet, JL et Normand,R., l'Europe de l'éducation : entre management et politique, ESEN-INRP, Lyon.

Bidwell, Charles, E. (1965), The school as a formal organization. In: Marsch James G., Handbook of Organisation, Rand MacNally and Company, Chicago.

Chatel, Elisabeth, 2002, Professeur de SES au lycée, un métier et un art, Hachette. 
Cooper, B., Fusarelli, L.D.et Vance-Randall, E., 2004, Better Policies, better Schools, Allyn \& Bacon, New York.

Darling-Hammond, L., 1990, Teacher Professionalism: Why and How? In Lieberman, A, Schools as collaborative cultures, Falmer Press, Bristol.

Derouet, JL, 1992, Ecole et justice, Métaillié, Paris.

Dutercq. Y., 2006, Les nouveaux pouvoirs des chefs d'établissement. In : Chapelle, G. et Meuret, D.(eds), Améliorer l'école, PUF, Paris, 141-147.

Duru-Bellat, M. et Meuret, D., 2002, Vers de nouveaux modes de gouvernement des systèmes éducatifs ? Les leçons des comparaisons internationales, Politiques et Management Public, 20 (2), 61-80.

Gather-Thurler, M., 2002, L'autoévaluation de l'établissement scolaire comme moteur du changement, in Bois, $\mathrm{M}$, dir., Les systèmes scolaires et leur régulation, CRDP, Lyon.

Grellier, Y., 1998, Profession, chef d'établissement, ESF, Paris.

Grisay, A. 1997, Evolution des acquis cognitifs des élèves au cours des années de collège, MEN-DEP, Dossiers Education et Formations, $n^{\circ} 88$.

Hassani, M., 2007, La régulation interne des établissements scolaires, Thèse de doctorat, Université de Bourgogne.

Kherroubi, M. et van Zanten, A., 2000, La coordination du travail dans les établissements difficiles : collégialité, division des rôles et encadrement, Education et Sociétés, $n^{\circ} 6$.

Lang, V., 1999, La professionnalisation des enseignants, PUF, Paris.

Leightwood, K. \& Menzies, T., 1998, A review of research on School Based Management, School Effectiveness and School Improvement, 9(3), 233-285.

Lessard, C. \& Meirieu, Ph., 2005, L'obligation de résultats en éducation, de Boeck, Bruxelles.

Louis, K.S. et al. (2005), State Mandated Accountability in High Schools: Teachers interpretation of a new Era, Educational Evaluation and Policy Analysis, 27(2), 177-204.

Mc Neil, L., 1986, Contradictions of Control: School culture and School Knowledge, Routledge, London.

Mc Neil, L., 2000, Contradictions of School Reform, Routlege, London.

Maroy, Ch., 1992, L'école à la lumière de la sociologie des organisations, Université de Louvain.

Maroy, Ch., 2005, Les évolutions du travail enseignant en France et en Europe, Cahiers du GIRSEF $\mathbf{n}^{\circ} \mathbf{4 2}$, Université de Louvain la Neuve.

Maroy, Ch., 2006, Ecole, Régulation et Marché, PUF, Paris.

Meuret, D., 2007, Gouverner l'école, PUF, Paris.

Meuret, D., 2007b, Une défense de la régulation anglo-saxonne : les expériences anglaise et américaine, in Derouet, JL et Normand, R., l'Europe de l'éducation : entre management et politique, ESEN-INRP, Lyon. 
Meuret, D., Duru-Bellat, M., 2003, English and French Modes of Regulation of the the Education System, a comparison, Comparative Education, 39(4), 463-477.

Meuret, D., Broccholochi, S., Duru-Bellat, M., 2001 Autonomie et choix des établissements scolaires : finalités, modalités, effets, Cahiers de l'IREDU, $n^{\circ}$ 62, Dijon.

Mintzberg, H. (1982), Structure et dynamique des organisations, Ed. d'Organisation, Paris.

Rayou, P. \& van Zanten, A., 2004, Enquête sur les nouveaux enseignants, Changeront-ils l'école ? Bayard, Paris.

Tardif, M. \& Lessard, C., 2004, La profession d'enseignant aujourd'hui : évolutions, perspectives et enjeux internationaux, Presses de I'Université Laval, Laval (Québec).

Valencia, R., 2004, Let's treat the cause, not the symptoms: equity and accountability revisited, in Skrla, L. \& Scheurich, JJ., Educational Equity and Accountability, Routledge, London, 29-39.

Cour des Comptes, 2007, Rapport Public Annuel, chapitre sur les Etablissements Publics d'enseignement du second degré, Paris.

Commission Pochard, 2008, Livre vert sur l'évolution du métier d'enseignant, disponible sur le site du Ministère de l'Education.

OCDE, 2007, Améliorer la direction des établissements scolaires, rapport sur la France, coordonné par J.P. Obin, OCDE, Paris. (disponible sur le web).

OCDE, 2008, Regards sur l'Education, OCDE, Paris. (disponible sur le web). 


\section{Annexe 1 - Résultats bruts du questionnaire}

Les questions, les modalités de réponse à chacune ainsi que le pourcentage (arrondi à l'unité) de l'ensemble des répondants ayant choisi chaque modalité sont indiqués ci-dessous. Par exemple, la présentation de la première question ( $q 1$ Dans votre établissement scolaire, combien de fois par an, en moyenne, les enseignants d'une même discipline se réunissent-ils?) se lit : $1 \%$ des chefs d'établissement qui ont répondu à cette question ont choisi la modalité 1, c'est-à-dire « jamais ", $5 \%$ ont choisi la modalité 2, c'est-à-dire " une fois », $27 \%$ ont choisi la modalité 3 , c'est-àdire « deux fois », 33\% ont choisi la modalité 4 (« trois fois ») et $33 \%$ aussi ont choisi la modalité 4 (plus de trois fois). Ces indications permettent de lire l'annexe 2 (par exemple, q11 y désigne ceux qui ont répondu la modalité 1 à la question 1). Le questionnaire comportait en outre quelques questions permettant de caractériser l'établissement ainsi que le répondant. Les questions sont ici regroupées selon les concepts utilisés dans l'article, elles étaient présentées en ordre numérique croissant dans le questionnaire. La réponse modale est en gras.

\section{Coopération entre enseignants}

q1. Dans votre établissement scolaire combien de fois par an, en moyenne, les enseignants d'une même discipline se réunissent-ils? 1 jamais : $1 \%, 2$ une fois : $5 \%$, 3 deux fois : $27 \%, 4$ trois fois : $33 \%, 5>3$ fois : $33 \%$

q4. Dans votre établissement scolaire combien de fois par an, en moyenne, les enseignants d'une même classe se réunissent-ils de façon formelle en dehors des conseils des classes ? 1 jamais : $5 \%, 2$ une fois : $16 \%, 3$ deux fois $29 \%$, 4 trois fois : $25 \%, 5$ >trois fois : $25 \%$

q6. Dans votre établissement, arrive-t-il que les enseignants prennent ensemble des décisions pédagogiques ? 1jamais :2\%,2 rarement : 7\%,3 quelque fois : $40 \%, 4$ assez souvent : $35 \%, 5$ souvent $: 16 \%$.

q8 Dans votre établissement, les enseignants d'une même classe se coordonnent-ils entre eux ? 1 jamais : $0 \%, 2$ rarement : $7 \%, 3$ quelquefois : $25 \%, 4$ assez souvent : $46 \%, 5$ souvent : $22 \%$.

q10. Dans votre établissement scolaire, les enseignants discutent-ils entre eux du niveau de performance de leurs élèves et des moyens d'améliorer la performance des uns et des autres ? 1 jamais : $1 \%, 2$ rarement : $6 \%, 3$ quelquefois : $18 \%, 4$ assez souvent : $40 \%, 5$ souvent : $35 \%$.

q11. Dans votre établissement y a-t-il une coordination entre les enseignants d'une même discipline sur l'objet ou les méthodes de l'évaluation? 1 jamais : $6 \%, 2$ rarement : $15 \%, 3$ quelquefois : $41 \%$, 4 assez souvent : $25 \%$, 5 souvent : $13 \%$.

q12. Les enseignants acceptent volontiers de participer aux activités de l'établissement 1NSP : 0\%, 2 en désaccord : 1\%, 3 plutôt en désaccord $14 \%, 4$ plutôt d'accord : $63 \%, 5$ tout à fait d'accord : $22 \%$.

q13. Dans mon établissement, les enseignants partagent les mêmes valeurs professionnelles. 1NSP : $2 \%$, 2 en désaccord : $4 \%$, 3 plutôt en désaccord 20\%, 4 plutôt d'accord : $65 \%$, 5 tout à fait d'accord : $9 \%$. 
q25. Diriez vous que, tous comptes faits, le travail collectif existe dans votre établissement 1 oui $86 \% 2$ non $14 \%$.

q26. Si oui, combien d'heures, en moyenne, chaque enseignant consacret-il à se coordonner avec les collègues de sa classe ou de sa discipline pendant un mois ? 1 une heure ou moins : $20 \%$, 2 deux heures : $32 \%, 3$ trois heures : $23 \%$, plus de trois heures : $12 \%$.

\section{Normes professionnelles des chefs d'établissement}

q 14. Travailler en équipe ajoute beaucoup à l'efficacité de chaque enseignant....1 NSP : $1 \%$, 2 en désaccord : $0 \%$, 3 plutôt en désaccord $0 \%$, 4 plutôt d'accord : $28 \%, 5$ tout à fait d'accord : $71 \%$.

q16.L'une des tâches du chef d'établissement est la direction et la supervision du travail des enseignants 1NSP : $3 \%$, 2 en désaccord : $5 \%, 3$ plutôt en désaccord $5 \%, 4$ plutôt d'accord : $51 \%, 5$ tout à fait d'accord : $27 \%$.

q17. Les enseignants d'une discipline doivent toujours avoir le dernier mot quant à la pédagogie de cette discipline $1 \mathrm{NSP}: 3 \%, 2$ en désaccord: 15\%, 3 plutôt en désaccord $37 \%, 4$ plutôt d'accord : $\mathbf{3 8} \%, 5$ tout à fait d'accord: $7 \%$.

q18. Les chefs d'établissements doivent inciter les enseignants à contrôler eux-mêmes la qualité de leur travail...1NSP : $1 \%$, 2 en désaccord : $2 \%, 3$ plutôt en désaccord $3 \%, 4$ plutôt d'accord : $61 \%, 5$ tout à fait d'accord: $34 \%$.

q19. Le chef d'établissement doit jouer un rôle pédagogique. 1NSP : 0\%, 2 en désaccord : $1 \%, 3$ plutôt en désaccord : $2 \%, 4$ plutôt d'accord : $21 \%, 5$ tout à fait d'accord : $76 \%$.

q20. Le contrôle de la qualité du travail des enseignants par le chef d'établissement est légitime. 1NSP : $0 \%$, 2 en désaccord : $1 \%, 3$ plutôt en désaccord $3 \%, 4$ plutôt d'accord : $36 \%, 5$ tout à fait d'accord : $61 \%$.

q21. La plupart des enseignants aiment être responsables et autonomes dans leur travail. 1NSP : 1\%, 2 en désaccord : $0 \%, 3$ plutôt en désaccord 0\%, 4 plutôt d'accord : $45 \%, 5$ tout à fait d'accord : $\mathbf{5 3} \%$.

q22. L'intervention du chef d'établissement sur le travail des enseignants améliorerait la qualité de leur travail 1NSP : $12 \%$, 2 en désaccord : $5 \%, 3$ plutôt en désaccord $12 \%, 4$ plutôt d'accord : $51 \%, 5$ tout à fait d'accord : $20 \%$.

q23. C'est d'abord le travail de tous les enseignants qui fait l'efficacité de mon établissement...1NSP : $1 \%$, 2 en désaccord : $0 \%, 3$ plutôt en désaccord $2 \%, 4$ plutôt d'accord : $38 \%, 5$ tout à fait d'accord : $59 \%$.

q24. D'après mon expérience, les réunions entre enseignants sont plutôt une perte de temps. 1 NSP : $2 \%$, 2 en désaccord : $71 \%$, 3 plutôt en désaccord 24\%, 4 plutôt d'accord : $3 \%, 5$ tout à fait d'accord : $1 \%$.

\section{Régulation des enseignants par le chef d'établissement}

q3. Combien de fois par an, en moyenne, entrez vous dans la classe d'un enseignant même de façon rapide et informelle? 1 jamais : $8 \%, 2$ une fois $10 \%, 3$ deux fois $10 \%, 4$ trois fois : $7 \%, 5>$ trois fois : $66 \%$. 
q5. II vous arrive de discuter avec les enseignants des points faibles et forts de leur travail... : 1 jamais : $2 \%$, 2 rarement : $15 \%$, 3 quelque fois : $39 \%, 4$ assez souvent : $33 \%, 5$ souvent : $12 \%$.

q7. Dans votre établissement, il vous arrive de conseiller à un enseignant de suivre telle formation continue ...

1jamais : $1 \%$, 2 rarement : $23 \%, 3$ quelque fois : $59 \%, 4$ assez souvent : $11 \%, 5$ souvent : $6 \%$.

q9. Rencontrez vous les enseignants pendant la récréation ou à d'autres occasions informelles, même de façon rapide? 1 jamais : $0 \%, 2$ rarement : $2 \%$, 3 quelque fois : $5 \%, 4$ assez souvent : $16 \%, 5$ souvent : $77 \%$.

q15. Confier telle classe de tel niveau scolaire à un enseignant se fait sur la base des résultats d'une évaluation (explicite ou implicite) de son efficacité. 1NSP : 1\%, 2 en désaccord : 4\%, 3 plutôt en désaccord 13\%, 4 plutôt d'accord : $53 \%$, 5 tout à fait d'accord : $29 \%$.

q27. Est-ce que vous contrôlez les cahiers de texte des enseignants ? 1 oui : $72 \%$, 2 non : $28 \%$.

q28. Si oui, en moyenne, combien de fois par an : 1 une fois $39 \%$, 2 deux fois : $22 \%, 3$ trois fois $9 \%, 4$ plus de trois fois : $2 \%$.

q29. Rencontrez-vous individuellement les enseignants pour discuter et évoquer les performances de leurs élèves ? 1 oui : $76 \%$, 2 non : $24 \%$.

q30. Si oui, combien de fois par an, en moyenne ? 1 une fois : $25 \%$, 2 deux fois : $18 \%, 3$ trois fois : $15 \% 4$ plus de trois fois : $16 \%$.

q31. Avez-vous indiqué aux enseignants les critères selon lesquels vous appréciez la qualité de leur travail ? 1 oui, de façon formelle : $27 \%, 2$ oui, de façon plutôt informelle : $60 \%$, 3 à certains, à certaines occasions : $11 \%, 4$ jamais : $3 \%$.

q35. L'année dernière, êtes vous intervenu dans la classe d'un enseignant à propos d'un problème rencontré par ce dernier ? 1 oui : 76\%, 2 non : $24 \%$. q36. si oui, combien de fois est ce arrivé ? 1 une fois 10\%, 2 deux fois : $29 \%, 3$ trois fois : $19 \%, 4$ plus de trois fois : $20 \%$.

q 37. Vous assurez vous, en tant que chef d'établissement, de l'adaptation du travail des enseignants aux objectifs et aux programmes de leur discipline? 1 oui : $64 \%$, 2 non : $36 \%$.

q38. Si oui, cela se réalise-t-il plutôt par l'examen des résultats qu'ils obtiennent avec leurs élèves ou par l'examen de ce qui se passe dans leur classe ? 1 par les résultats 24\%, 2 par l'examen de la classe : $29 \%$, 3 les deux : $11 \%$.

q39. En moyenne, combien de fois par semaine rencontrez vous les enseignants dans la salle des professeurs ?1 une fois ou moins : $12 \%, 2$ deux fois : $9 \%, 3$ trois fois : $12 \%$, plus de trois fois : $67 \%$.

q40. Selon vous, votre action a-t-elle sur l'enseignement en classe, une influence... 1 forte : $6 \%, 2$ assez forte : $58 \%$, 3 faible : $34 \%$, 4 nulle : $2 \%$.

\section{Pratiques de pilotage de l'établissement}

q2. Combien de fois par an réunissez-vous les enseignants pour parler du projet d'établissement ou de l'établissement en général? 1 jamais : $0 \%, 2$ une fois : $5 \%$, 3 deux fois : $36 \%$, 4 trois fois : $27 \%, 5>$ trois fois : $32 \%$. 
q32. Utilisez vous les indicateurs IPES (Indicateurs de Pilotage des Etablissements Secondaires, édités par la DEPP à l'intention de chaque lycée ou collège, public ou privé, du pays) pour évaluer votre établissement ? 1. régulièrement : $47 \%$, 2 exceptionnellement : $29 \%$, 3 non : $24 \%$.

q33. Si oui, discutez vous avec certains enseignants de ces indicateurs ? 1 assez souvent $22 \%$, 2 quelque fois : $23 \%$, 3 rarement : $5 \%, 4$ jamais : $6 \%$. q34. Utilisez vous les indicateurs de l'Inspection Académique ou du Rectorat pour évaluer votre établissement ? 1 régulièrement : $69 \%, 2$ exceptionnellement : $31 \%$, 3 non : $13 \%$. 
Annexe 2 - Classification hiérarchique des réponses

Présentation des réponses dans les deux premiers plans factoriels

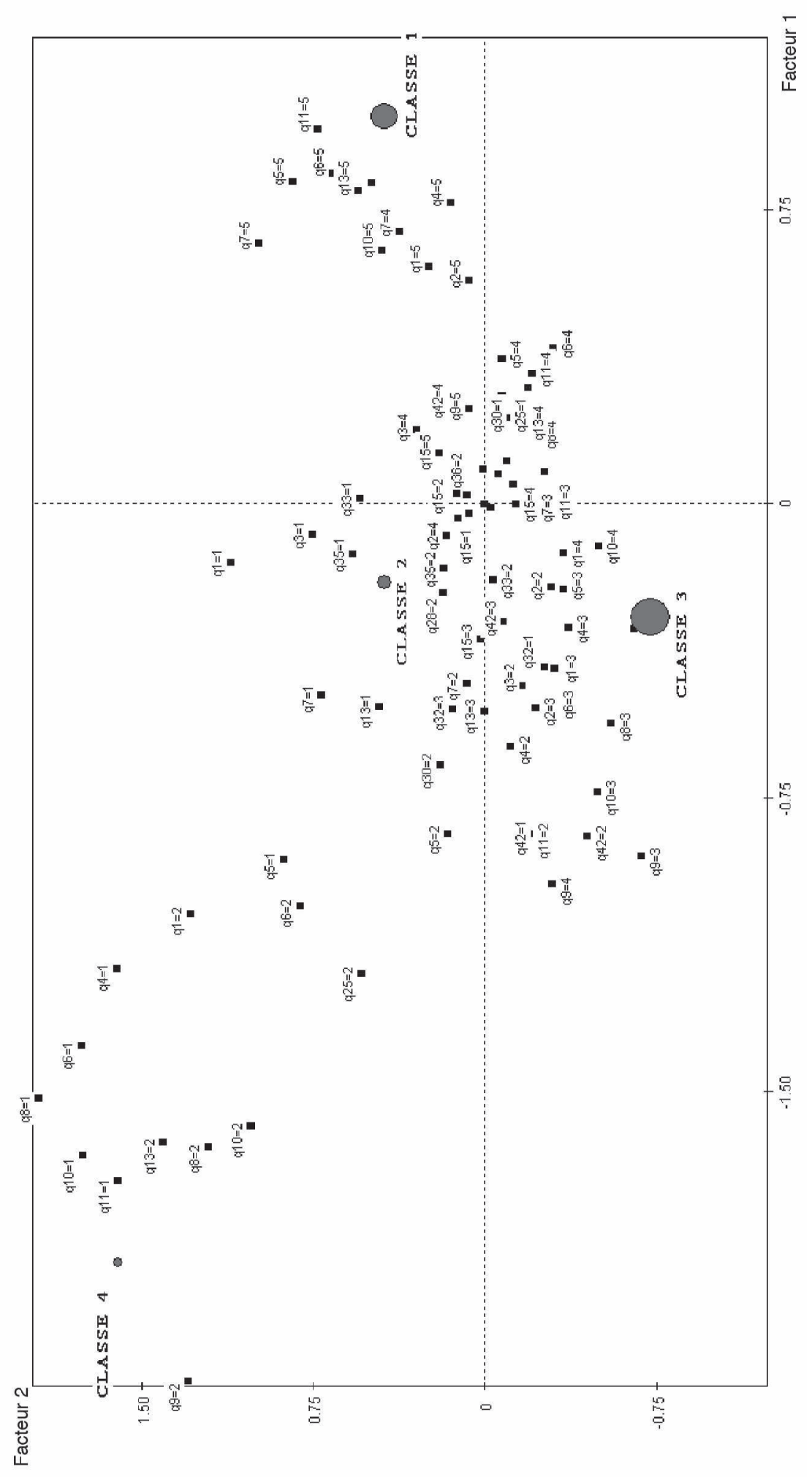


